https://doi.org/10.15407/ujpe66.7.588

A. KUMAR, ${ }^{1}$ D.P. OJHA ${ }^{2}$

${ }^{1}$ Faculty of Sciences (Physics), National Defence Academy

(Khadakwasla, Pune-411023, Maharashtra,India; e-mail: ashuphys@gmail.com)

2 School of Physics, Sambalpur University

(Jyoti Vihar, Burla, Sambalpur, Odisha-768019, India)

\title{
STUDIES OF THERMODYNAMICAL AND ELECTRONIC TRANSPORT PROPERTIES OF Na-Sn ALLOY
}

\begin{abstract}
The concept of complex formation has been incorporated in the structure of the Faber-Ziman formula for the purpose of studying the composition dependence of the electrical resistivities of Zintl alloys, which possess the anomalous nature and exhibit a large deviation from the metallic behavior around a specific composition.
\end{abstract}

Ke ywords: mixing, fluids, electronic transport, liquid alloys, thermodynamic properties.

\section{Introduction}

In view of the widespread practical applications, the physical properties of liquid binary alloys have attracted the attention of both theoreticians and experimentalists for past several decades [1-10]. A typical example is the liquid alkali-IV group element alloys, i.e., Zintl alloys, which are found to exhibit a quite peculiar crystal structure [11] and the anomalous behavior of structural properties, electronic structure, and thermodynamic properties. The calculations by Geertsma et al. [12] show that there is the nonclustered-to-clustered transition in Zintl alloys. Such transition is viable due to the size effect that may be supported by the fact that the asymmetric behavior of the alloy is usually attributed $[13,14]$ to the interaction between solute and solvent atoms or both. The role of alkali atoms is mainly to keep anions apart with the loss of the valence electron. The anions with valence electrons are responsible for the metallic-covalent part of the bonding, and the electrical conductivity is basically due to the electron hopping from one tetrahedron to another one. Thus, a low value of the anion-anion interaction between two adjacent clusters corresponds to a high resistivity, while a large anion-anion interaction within a cluster corresponds to a clustered configuration. As the size of alkali atoms increases, the anions are pushed so far apart that the anion-anion interaction between two adjacent clusters decreases considerably.

(C) A. KUMAR, D.P. OJHA, 2021
It is, therefore, more energetically favorable for the anions to group together, and the resistivity increases [15]. Such ionic compounds form the subject of the research interest that seeks to explain their valences in terms of the charge transfer. The variation of the alloy composition allows a change of the dominant type of interaction from metallic to ionic and to partly covalent due to the charge transfer. This leads, e.g., to a strong concentration dependence of the thermodynamic and electronic transport properties [16]. The electrical conduction theory in the liquid metal using the pseudopotential concept provides an alluring field for investigating the electron transport properties of liquid metals and alloys, as they are used in many fields of engineering and in metallurgical processes.

In the present communication, an attempt has been made to study the thermodynamical property. We will consider Gibbs' function based on the concept of the complex formation model (CFM) and the structure of Faber-Ziman's [17] formula as the most popular interpretation of anomalous thermodynamic data. We will assume the existence of chemical complexes (or associates) with finite lifetime, although there is no direct evidence for them. However, the indication for such type of preferential association of the constituent species of the alloy can be found in their phase diagrams and in X-ray and neutron diffraction patterns. The computations have been carried out for all the properties at $773 \mathrm{~K}$ based on the experimental data available for thermodynamical properties [18].

ISSN 2071-0194. Ukr. J. Phys. 2021. Vol. 66, No. 7 


\section{Theoretical Methodology}

\subsection{Thermodynamical properties}

In the complex formation, all mixed atoms are not in random arrangement. Some of them are randomly distributed, while others coalesce into small groups bound by covalent bonds. Hence, the liquid metal binary alloy becomes a ternary system consisting of free constituent ions of the component metals and chemical complexes. The complex formation model $[2,13,19-24]$ assumes the liquid binary alloy $A-B$ as a ternary mixture consisting of free atoms $A$, free atoms $B$, and their preferential associates referred as chemical complexes $A_{\mu} B_{\nu}$.

Let us suppose that $n_{A}\left(=n_{1} N\right)$ of free atoms $A$, $n_{B}\left(=n_{2} N\right)$ of free atoms $B$, and $n_{m}\left(=n_{3} N\right)$ number of chemical complexes exist in the mixture. Then the total number of scattering points is

$N_{S}=n_{A}+n_{B}+n_{m}=n N$,

where $N$ is the total number of atoms $A$ and atoms $B$, i.e., $N=N_{A}+N_{B}$ and $n=n_{1}+n_{2}+n_{3}$ with

$n_{1}=1-C-\mu n_{3}$,

$n_{2}=C-\nu n_{3}$

where $C$ is the concentration of the second component.

The free energy of the mixing $G_{M}$ (Gibbs' energy or Gibbs' function) of the mixture may be written as

$G_{M}=-n_{3} g+G^{1}$,

where $g$ is the formed complex energy. Thus, the first term $-n_{3} g$ represents the lowering of the free energy due to the formation of the complex in the alloy. $G^{1}$ is the free energy of mixing of the ternary mixture $A, B$ and $A_{\mu} B_{\nu}$. Since strong interactions are taken care of, via the formation of chemical complexes, the mixture can be treated as weakly interacting system. Hence, for $G^{1}$, the conformal solution approximation [25] can be considered. This enables us to express $G_{M}$ as

$$
\begin{aligned}
& G_{M}=-n_{3} g+R T \sum_{i=1}^{3} n_{i}\left(\ln n_{i}-\ln n\right)+ \\
& +\sum_{i<j} \sum \frac{n_{i} n_{j}}{n} W_{i j} .
\end{aligned}
$$

Here, $W_{i j}(i, j=1,2,3)$ are the interaction energies, $R$ is the molar gas constant, and $n$ is the total number of atoms in the case of compound formation.
The equilibrium values of the chemical complex $n_{3}$ may be obtained through the condition

$$
\begin{aligned}
& \left(\frac{\partial G_{M}}{\partial n_{3}}\right)_{T, P, C}=0, \\
& \frac{n_{1}^{\mu} n_{2}^{\nu}}{n_{3} n^{\mu+\nu-1}}=e^{-g / R T} e^{Y},
\end{aligned}
$$

where

$$
\begin{aligned}
& Y=\frac{W_{12}}{R T}\left[(\mu+\nu-1) \frac{n_{1} n_{2}}{n^{2}}-\frac{\mu n_{2}}{n}-\frac{\nu n_{1}}{n}\right]+ \\
& +\frac{W_{13}}{R T}\left[(\mu+\nu-1) \frac{n_{1} n_{3}}{n^{2}}-\frac{\mu n_{3}}{n}+\frac{n_{1}}{n}\right]+ \\
& +\frac{W_{23}}{R T}\left[(\mu+\nu-1) \frac{n_{2} n_{3}}{n^{2}}-\frac{\nu n_{3}}{n}+\frac{n_{2}}{n}\right] .
\end{aligned}
$$

The equation can be solved numerically to obtain the equilibrium value of $n_{3}$.

The work has been initiated with the presumption that the chemical complex NaSn is formed in $\mathrm{Na}^{-}$ Sn alloy. The interaction energies $W_{i j}$ and $g$ have been computed with the procedure suggested by Bhatia and Hargrove [19]. The value of $g$ has been taken nearly equal to $-(\mu+\nu) G_{M}$ as a starting point. Then the interaction energies $W_{12}, W_{13}$ and $W_{23}$ have been adjusted to get the concentrationdependent free energy of mixing $G_{M}$ through Eqs. [4] and [5]. The process has been repeated for different sets of energy parameters until a good fit for $G_{M}$ is obtained. It may, however, be noted that once the energy parameters have been selected, they remain the same for all mixing.

\subsection{Electronic transport properties}

The computation of the electrical resistivity of ternary systems requires three scattering potentials (form-factors) corresponding to two- component metals and chemical complexes and six partial structure factors [2]. The model parameters obtained by Ese and Reissland, 1973 [26] shown in Table 1 have been used to determine the form-factors. Incorporating the CFM in the structure of the Faber-Ziman [17] formula, the electrical resistivity of the compoundforming binary alloy may be expressed as

$R=R_{1}+R_{2}$,

where $R_{1}$ and $R_{2}$ represent, respectively, the contributions from the scattering by bare $A$ and $B$ ions 
and from the chemical complex $A_{\mu} B_{\nu}$ which can be expressed as

$R_{1}=\frac{3 \pi}{\hbar e^{2}} \frac{\Omega}{v_{\mathrm{F}}^{2}}\left\langle F_{1}(q)\right\rangle$,

$R_{2}=\frac{3 \pi}{\hbar e^{2}} \frac{\Omega}{v_{\mathrm{F}}^{2}}\left\langle F_{2}(q)\right\rangle$,

where $\Omega$ is the volume of the alloy, $v_{\mathrm{F}}$ is the Fermi velocity,

$\left\langle F_{1}(q)\right\rangle=4 \int_{0}^{1}\left[x_{1} w_{1}^{2}(\mathbf{k}, \mathbf{q}) S_{11}(K)+x_{2} w_{2}^{2}(\mathbf{k}, \mathbf{q}) S_{22}(K)+\right.$

$\left.+2\left(x_{1} x_{2}\right)^{1 / 2} w_{1}(\mathbf{k}, \mathbf{q}) w_{2}(\mathbf{k}, \mathbf{q}) S_{12}(K)\right] \eta^{3} d \eta$,

$\left\langle F_{2}(q)\right\rangle=4 \int_{0}^{1}\left[x_{3} w_{3}^{2}(\mathbf{k}, \mathbf{q}) S_{33}(K)+2\left(x_{2} x_{3}\right)^{1 / 2} w_{2}(\mathbf{k}, \mathbf{q}) \times\right.$

$\times w_{3}(\mathbf{k}, \mathbf{q}) S_{32}(K)+2\left(x_{1} x_{3}\right)^{1 / 2} \times$

$\left.\times w_{1}(\mathbf{k}, \mathbf{q}) w_{3}(\mathbf{k}, \mathbf{q}) S_{31}(K)\right] \eta^{3} d \eta$.

Here, $x_{1}, x_{2}$, and $x_{3}$ are, respectively, the concentration fraction of three scattering centers $A, B$, and $A_{\mu} B_{\nu}$ which can further be expressed as

$x_{1}=\frac{n_{A}}{N_{S}}, x_{2}=\frac{n_{B}}{N_{S}}$ and $x_{3}=\frac{n_{m}}{N_{S}}$.

The quantities $S(K)$ and $w(k, q)$ with subscripts 1,2 , 3 represent, respectively, the partial structure factors and non-local screened form-factors of the constituent elements and a chemical complex.

The values of the screened form-factor $w_{i}(k, q)$ as a function of the compositions have been obtained using the energy-dependent non-local optimized model potential of Shaw [27], because it has proved very successful in the study of electronic transport properties

Table 1. Model parameters (a.u. = 2 Ryd.) and their derivatives at the Fermi energy

\begin{tabular}{|c|c|c|c|c|c|c|}
\hline Metal & $a_{0}$ & $a_{1}$ & $a_{2}$ & $\delta a_{0} / \delta E$ & $\delta a_{1} / \delta E$ & $\delta a_{2} / \delta E$ \\
\hline $\mathrm{Na}$ & 0.312 & 0.357 & - & -0.187 & -0.117 & - \\
\hline $\mathrm{Sn}$ & 1.831 & 2.052 & 1.795 & -0.478 & -0.246 & 0.183 \\
\hline Compound & $a_{0}$ & $a_{1}$ & $a_{2}$ & $\delta a_{0} / \delta E$ & $\delta a_{1} / \delta E$ & $\delta a_{2} / \delta E$ \\
\hline $\mathrm{NaSn}$ & 2.143 & 2.40 & - & -0.38 & -0.18 & - \\
\hline
\end{tabular}

of liquid metals. The screened non-local pseudopotential matrix elements can be expressed as

$$
\begin{aligned}
& w_{i}(k, q)=f_{i}(K, q)+\frac{4 \pi}{\Omega q^{2} \varepsilon^{*}(q)} \times \\
& \times\left[1-G(q) \rho_{i}+g_{i}(q)\left(1-\frac{G(q)}{\varepsilon^{*}(q)}\right)\right],
\end{aligned}
$$

with

$f_{i}(\mathbf{K}, \mathbf{q})=-N \sum_{l=0}^{l_{0}}\left\langle\mathbf{k}+\mathbf{q}\left|\left(A_{i l}(E)-\frac{Z_{i}}{r}\right) P_{l}\right| \mathbf{k}\right\rangle=$ $=-\frac{4 \pi}{\Omega} \sum_{l=0}^{l_{0}}(2 l+1) P_{l}(\cos \theta) \int_{0}^{r_{m}}\left(A_{i l}-\frac{Z_{i}}{r}\right) \times$

$\times j_{l}(|\mathbf{k}+\mathbf{q}| r) j_{l}(|\mathbf{k}|) r^{2} d r$.

Here, $j_{l}(x)$ are the spherical Bessel functions, $P_{l} \cos \theta$ are Legendre's polynomials, $g(q)$ is the non-local screening contribution given by

$$
\begin{aligned}
& g_{i}(q)=\frac{4}{\pi^{2} q^{2} \varepsilon^{*}(q)} \int_{K \leq K_{\mathrm{F}}} \frac{f_{i}(K, q)}{K^{2}-|K+q|^{2}} d^{3} K \\
& \rho_{i}=\frac{4}{\pi} \sum_{l=0}^{l_{0}}(2 l+1) \int_{0}^{K_{\mathrm{F}}} K^{2} d K \int_{0}^{r_{m}} j_{l}^{2}(K r)\left(\frac{\delta A_{i l}}{\delta E}\right) r^{2} d r,
\end{aligned}
$$

$K$ is the electron wave vector, $\rho_{i}$ is the depletion hole which has been determined following the Shaw procedure [27], and $l_{0}$ is the highest angular momentum to be modeled. For a given angular momentum, the energy-dependent model parameters $A_{i l}$ have been determined by matching the logarithmic derivative of the wave function at the boundary for different ionic term values which yield, in turn, crystal parameters at $E=E_{\mathrm{F}}$.

The electrical resistivity has been calculated on the assumption that $2 n_{m}$ valence electrons contribute to the formation of the chemical complex and, therefore, are localized in it. The number of conduction electrons $n_{C}$ moving in the alloy is given by

$n_{C}=\left[x_{1} Z_{1}+x_{2} Z_{2}\right] N-2 n_{m}$,

and the mean number of valence electrons per atom is

$Z_{S}=\left[x_{1} Z_{1}+x_{2} Z_{2}\right]-2 n_{m} / N$,

$K_{\mathrm{F}}=\left(\frac{3 \pi^{2} Z_{S}}{\Omega}\right)^{1 / 3}$.

ISSN 2071-0194. Ukr. J. Phys. 2021. Vol. 66, No. 7 
The experimental data on the partial and total structure factors of compound-forming binary alloys are unavailable with respect to compositions; in addition, they have insufficient accuracy. Thus, one has to divulge into the computational effort, and this is achieved through the hard-sphere reference system, obtaining the solutions of Percus-Yevik equation for an $m$-component hard sphere mixture $[28,29]$. The total structure factor $S(K)$ is given by

$$
\begin{aligned}
& S(K)=x_{1} S_{11}(K)+x_{2} S_{22}(K)+x_{3} S_{33}(K)+ \\
& +2\left(x_{1} x_{2}\right)^{1 / 2} S_{12}(K)+2\left(x_{1} x_{3}\right)^{1 / 2} S_{13}(K)+ \\
& +2\left(x_{2} x_{3}\right)^{1 / 2} S_{23}(K),
\end{aligned}
$$

where $S_{i i}(K)$ and $S_{i j}(K)$ are the partial structure factors, $\mathrm{cc}_{i}$ 's are the concentration fractions of the three scattering centers $A, B, A_{\mu} B_{\nu}(A=\mathrm{Na}, B=$ $=\mathrm{Sn}, \mu=1$ and $\nu=1)$. Here, $q$ is the wave vector.

These computations need two ingredients, namely, hard sphere diameters $\sigma_{i}$ and the packing fraction related by

$\eta=\pi(6 \Omega)^{-1} \sum x_{i} \sigma_{i}^{3}$.

Here, $x_{i}$ are the concentrations of the species, and $\Omega$ is the volume of the alloy.

The hard sphere diameters $\sigma_{1}$ and $\sigma_{2}$ are calculated by matching the first peak of the structure factors of the constituent elements at their melting temperature. They have been assumed to be independent of the temperature and concentration, and $\sigma_{3}$ have been taken as $8.2 \mathrm{~nm}$ for $\mathrm{Na}-\mathrm{Sn}$ alloy. The values of a hard sphere diameter of $\mathrm{Na}-\mathrm{Sn}$ alloy have been tabulated in Table 2. The number of complexes $n_{3}$ in conjunction with the hard sphere diameters are used to compute the partial and total structure factors.

\section{Results and Discussions}

In the present work, the concept of CFM has been incorporated in the structure of the well-known FaberZiman formula for the purpose of studying the electrical resistivities of a Zintl alloy as a function of the composition which possess the anomalous nature close to the stoichiometric composition.

The Faber-Ziman formula which holds for the weak scattering might prove seriously inadequate for the purpose, because the mean free path of the conduction electrons is reduced to its minimum value, which is the atomic distance, at the composition close to their resistivity maxima. However, one can think to investigate the applicability of the Faber-Ziman formula for the computations of the electrical resistivities of complex-forming liquid alloys with the presumption that the spin-orbit interaction, due to the close association of the constituent species of the alloy counterbalances the effect of a low mean free path.

\subsection{Thermodynamic Properties}

The phase diagram of liquid $\mathrm{Na}-\mathrm{Sn}$ alloy shows several intermetallic compounds in the solid state. But there is an indication of peaks at $\mathrm{Na}_{3} \mathrm{Sn}$ and NaSn. The neutron diffraction results for $\mathrm{Na}_{4} \mathrm{Sn}_{3}$ exhibit the most pronounced prepeak. In view of this, the work has been initiated in the case of $\mathrm{Na}-\mathrm{Sn}$ alloy with the presumption that the chemical complexes NaSn coexist with free $\mathrm{Na}$ atoms and free $\mathrm{Sn}$ atoms in liquid $\mathrm{Na}-\mathrm{Sn}$ alloys. The interaction energies obtained through the model are $W_{12}=-0.04$, $W_{13}=-0.56, W_{23}=-0.5$, and $g=4.2$ at $773 \mathrm{~K}$ (in terms of RT). On experimental grounds, a higher value of $g>3.5$ suggests that $\mathrm{Na}-\mathrm{Sn}$ is a strongly interacting system in the light of the formation energy $g$, and it comes in the category of the $\mathrm{Hg}-\mathrm{Na}$, $\mathrm{Hg}-\mathrm{K}, \mathrm{Tl}-\mathrm{Te}, \mathrm{Mg}-\mathrm{Bi}$, etc., for which the formation energies are much larger i.e., 8.294, 9.965, 10.84, and 16.7, respectively, in contrast to the light interacting systems like $\mathrm{Mg}-\mathrm{Sn}, \mathrm{Ag}-\mathrm{Al}$, and $\mathrm{Cu}-\mathrm{Sn}$. Further, we observe that all the interaction energies are repulsive in nature. This also supports the strongly interacting nature of $\mathrm{Na}-\mathrm{Sn}$ alloy.

The variations of computed values of Gibbs' function along with their experimental values and the equilibrium values $n_{3}$ of the complex as a function of Gibbs' function composition of the constituent

Table 2. Hard sphere diameters (a.u.)

of $\mathrm{Na}$ and $\mathrm{Sn}$ in $\mathrm{Na}-\mathrm{Sn}$ alloys $\sigma_{3}=8.2, \mathrm{~T}=773 \mathrm{~K}$

\begin{tabular}{|c|c|c|c|}
\hline $\begin{array}{c}\text { Conc. of } \\
\mathrm{Na} \mathrm{C}_{\mathrm{Na}}\end{array}$ & $\begin{array}{c}\text { Volume } \\
\Omega\end{array}$ & $\sigma_{\mathrm{Li}}$ & $\sigma_{\mathrm{Sn}}$ \\
\hline 1.0 & 277.2 & 6.16 & - \\
0.8 & 260.0 & 6.08 & 5.53 \\
0.6 & 237.0 & 6.06 & 5.57 \\
0.5 & 232.0 & 6.07 & 5.63 \\
0.4 & 225.6 & 6.06 & 5.66 \\
0.2 & 208.4 & 6.043 & 5.67 \\
0.0 & 191.2 & - & 5.73 \\
\hline
\end{tabular}




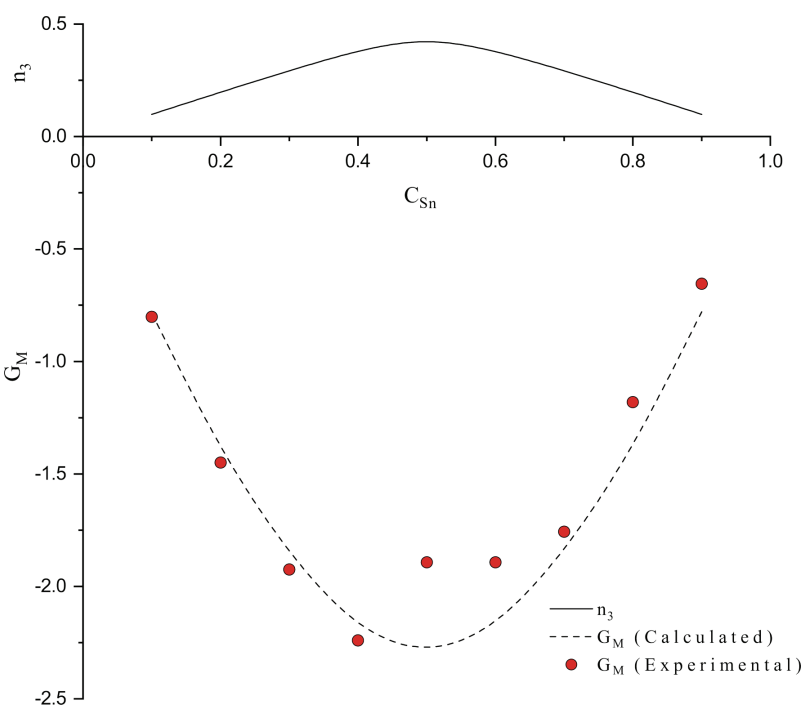

Fig. 1. Variation of Gibbs' function as a function of the composition at $773 \mathrm{~K}$

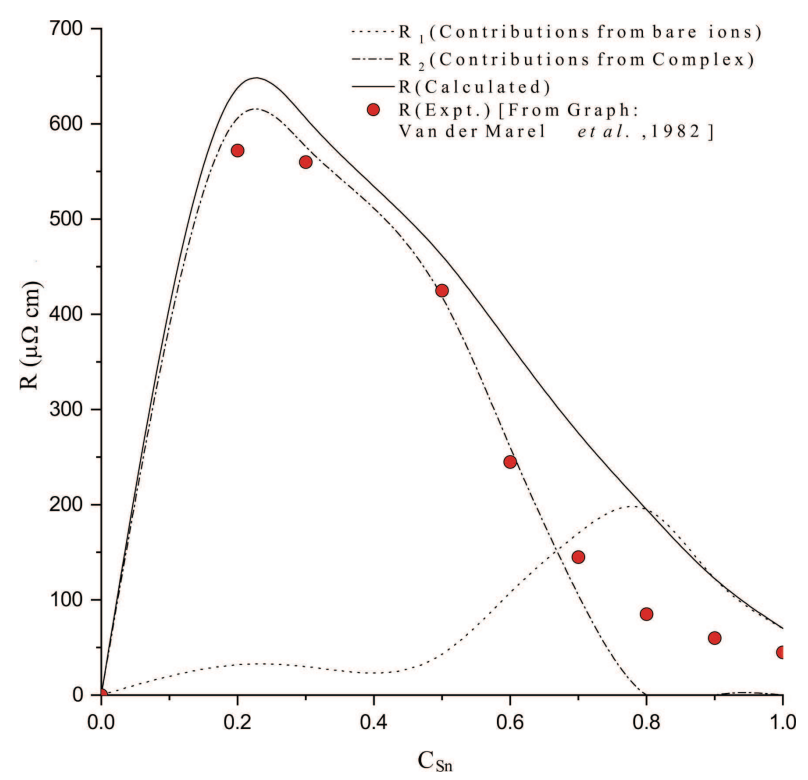

Fig. 2. Variation of the electrical resistivity of NaSn compound with respect to the concentration at $773 \mathrm{~K}$

species of the alloy have been shown in Fig. 1. The Gibbs' function $G_{M}$ shows a good agreement with experiment. We observe that $G_{M}$ is symmetric for $\mathrm{Na}^{-}$ $\mathrm{Sn}$ in contrary to systems $\mathrm{Li}-\mathrm{Sn}, \mathrm{Al}-\mathrm{Ca}, \mathrm{Ca}-\mathrm{Mg}, \mathrm{Mg}-$ $\mathrm{Zn}, \mathrm{Cu}-\mathrm{Mg}$, and so on which possess the asymmetry.

\subsection{Electronic Transport Properties}

The computed values of the electrical resistivity of compound NaSn have been shown in Fig. 2. The elec-

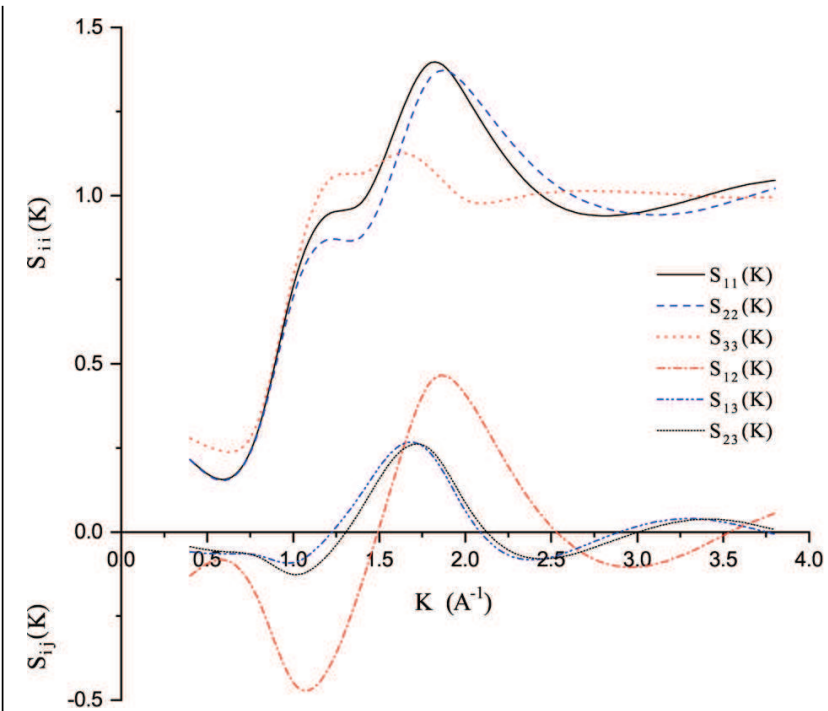

Fig. 3. Partial Structure factors of liquid NaSn compound of $\mathrm{Na}-\mathrm{Sn}$ Alloy at $773 \mathrm{~K}$

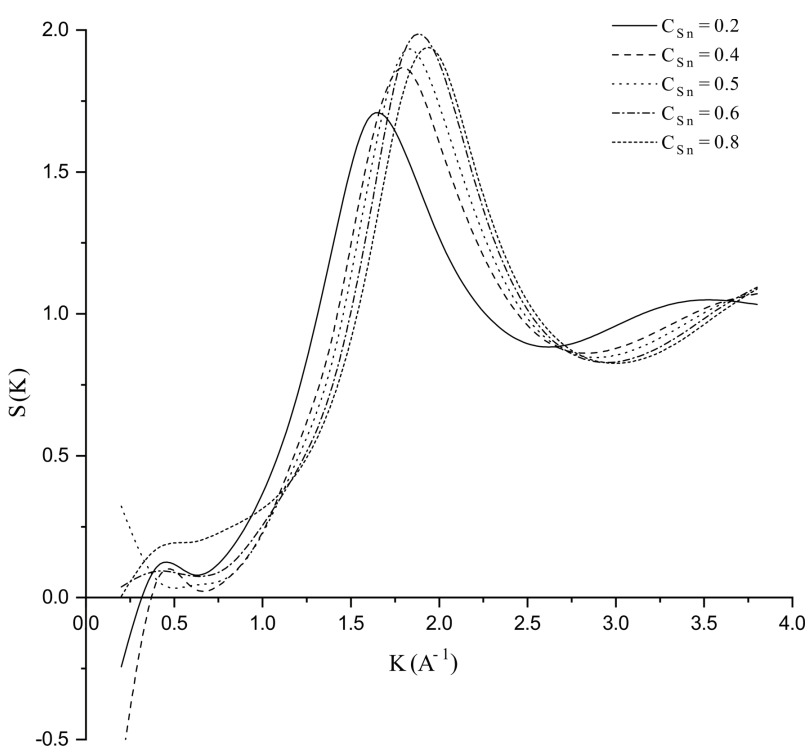

Fig. 4. Variation of the total structure factor with respect to the concentration of liquid NaSn compound of Na-Sn alloy at $773 \mathrm{~K}$

trical resistivity of the system is found to be asymmetric. No anomalous value is obtained at any concentration. The contributions from complexes have been found quite asymmetric having a sharp peak. The calculated value has been compared with the result of Van der Marel et al. [30]. An excellent agreement is found between the calculated and experimental values. The scattering contributions $\mathrm{R}_{1}$ from bare ions 
and the scattering contributions $\mathrm{R}_{2}$ from complexes have also been presented in the figure. The computed values of the electrical resistivities have been found in a close agreement with the experimental values.

The computed values of partial structure factors (PSF) of $\mathrm{Na}-\mathrm{Sn}$ which have been considered as ternary systems using the determined hard sphere diameters (Table 2) and equilibrium values $n_{3}$ of the complex at the composition, where the electrical resistivities have been found maximum, have been shown in Fig. 3. The PSF of unlike species are oscillatory about zero in this system. The magnitude of peak values of $S_{11}=1.3848$ have been found larger than that of $S_{22}=1.2858$ and $S_{33}=1.176$. The computed values of the total structure factors of liquid $\mathrm{Na}-\mathrm{Sn}$ alloy are shown in Fig. 4. As a consequence of the large difference in electronegativities between the constituent elements, the alloy tends to have salt-like properties. Such ionic compounds form the subject of chemical studies that seek to explain their valences in terms of the charge transfer.

It is found in Fig. 4 that the main peak of the PSF is shifting toward higher $K$ with the amount of doping of Sn atoms. This feature may be attributed to the charge transfer taking place in the system. The transition from the metallic to ionic regime is accompanied by a considerable volume contraction [31] due to shorter interatomic distances, which is reflected by a shift of the principal peak of $S(K)$ and of the dispersion minimum to larger $K$. The formation of strong $\mathrm{Na}-\mathrm{Sn}$ bonds also causes a shift of the dispersion, still dominated by the $\mathrm{Li}-\mathrm{Li}$ correlations, toward higher frequencies.

It should be mentioned that the PSF, $S(K)$, shows the behavior of a random mixing without a subpeak or asymmetry of the first peak and shows the compound-forming behavior with a sub-peak below the first peak [32]. The partial structure factors of unlike atom pairs have maxima, which lie in between those of like pair of atoms in the case of random mixing. On the other hand, in compound-forming alloys, the partial structure factor of unlike atom pairs has a very sharp peak with a sub-peak below the main peak which can also be seen in the figure. Various alloys also show their behavior in between these two types. The negative peak in the lower region of $k / k_{\mathrm{F}}$ indicates the preference for unlike nearest neighbors. The humps in the lower $\mathrm{K}$ regions are due to the short-range order (SRO) with the preference for unlike nearest neighbors [33]. Positive values of the partial structure factors imply that the repulsive core part of the effective interionic potential is dominating, whereas the negative values imply that the dominating part is attractive in nature.

\section{Conclusions}

The hard sphere reference system can be used to predict the atomistic structure of complex-forming alloys using the Hiroike exact solution of the Percus-Yevick equation for the $m$-component mixture.

1. The Faber-Ziman formula in conjunction with the complex formation model $(\mathrm{CFM})$ can be used in describing the electronic behavior of liquid alloys which possess anomalous nature.

2. The electrical resistivity has been calculated on the assumption that $2 n_{m}$ valence electrons contribute to the formation of the chemical complex and, therefore, are localized in it.

3. Substantial effect of equilibrium values $n_{3}$ of the complex on the structure factors of liquid $\mathrm{Na}-\mathrm{Sn}$ alloy has been found. The position of the peak values has been found shifted toward the longer wavelength side with an increase in the value of $n_{3}$.

4. Scattering contributions to Ziman's integrand from unlike scattering centers have been found oscillatory about zero, and their net effect seems to be negligible. Major scattering contributions to Ziman's integrand come from the complex-complex structures. The scattering contributions from bare ions have been found nearly asymmetric.

5. The anomalous electronic behavior of liquid $\mathrm{Na}-$ Sn alloy occurs due to the preferential ordering of unlike atoms.

6. The sub-peak prior to the first distinct peak in the PSF of like as well unlike atom pairs indicates the formation of a compound in this system.

7. The shoulder at the high wave-vector side of the main peak reflects the short $\mathrm{Sn}-\mathrm{Sn}$ distances characteristic of a tetrahedron. This shoulder may be interpreted as an evidence of the covalent bonding between some atoms in the liquid state.

8. The charge transfer takes place in the system, when $\mathrm{Sn}$ is added to the liquid Li.

The financial support rendered by the DST, CSIR, UGC, City place New Delhi and DAE-BRNS, Mumbai is gratefully acknowledged. 
1. F. Springelkamp, R.A. de Groot, placeW. Geertsma, W. van der Lugt, F.M. Mueller. Electronic structure of semiconducting $\beta$-NaSn. Phys. Rev. B 32, 2319 (1985).

2. A. Kumar, D.P. Ojha. Study of electronic density of states: Zintl alloys. Met. Mat. Trans. B 55, 362 (2011).

3. Q. Fang, H. Wendt. Performance and thermodynamic properties of $\mathrm{Na}-\mathrm{Sn}$ and $\mathrm{Na}-\mathrm{Pb}$ molten alloy electrodes for alkali metal thermoelectric converter (AMTEC). J. Appl. Electrochem. 26, 343 (1996).

4. Y. Satoshi, O.I. Takno, H. Satoru. Observation of lithium isotope effect accompanying electrochemical insertion of lithium into tin. J. Nucl. Sci. and Tech. 37, 919 (2000).

5. O. Genser, J. Hafner. Structure and bonding in crystalline and molten Li-Sn alloys: A first-principles densityfunctional study. Phys. Rev. B 63, 144204 (2001).

6. Y. Kang, T. Terai. In-reactor experiment and the tritium diffusion coefficient in molten lithium-tin alloy. J. Nucl. Mater. 329-333, 1318 (2004).

7. placeS. Jahn, J.-B Suck, M.M. Koza. Atomic dynamics in liquid $\mathrm{K}_{x} \mathrm{Sb}_{1-x}$ alloys. J. Non Cryst. Sol. 353, 3145 (2007).

8. A. Thakur, P.K. Ahluwalia. Electrical resistivity of NaSn compound forming liquid alloy using ab initio pseudopotentials. Physica B 373, 163 (2006).

9. M. Saboungi, W. Geertsma, D.L. Price. Ordering in liquid alloys. Annu. Rev. Phys. Chem. 41, 207 (1990).

10. O. Akinlade. Ordering phenomena in $\mathrm{Na}-\mathrm{Ga}$ and $\mathrm{Na}-\mathrm{Sn}$ molten alloys. Phys. Chem. Liq. 29, 9 (1995).

11. H. Schaefer, B. Eisenmann, W. Muller. Zintl phases: Transitions between metallic and ionic bonding. Angew. Chem. 12, 694 (1973).

12. placeW. Geertsma, J. Dijkstra, W. van der Lugt. Electronic structure and charge-transfer-induced cluster formation in alkali-group-IV alloys. J. Phys. F.: Met. Phys. 14, 1833 (1984).

13. A.B. Bhatia. Proc. $3^{\text {rd }}$ Int. Conf. on Liquid Metals, 1976 (Inst. Phys. of Bristol, 1977), Vol. 30, p. 21.

14. R.N. Singh. Short-range order and concentration fluctuations in binary molten alloys. Can. J. Phys. 65, 309 (1987).

15. B. Wang. Ab Initio Studies of Pure Sn and Mixed $N a-S n$ Clusters with Implications for Liquid $\mathrm{Na}-\mathrm{Sn}$ Alloys. $\mathrm{PhD}$ Thesis (Queen's University, 1997).

16. W. van der Lugt. Polyanions in liquid ionic alloys: A decade of research. J. Phys. Condens. Matter. 8, 6115 (1996).

17. T.E. Faber, J.M. Ziman. A theory of the electrical properties of liquid metals. Phil. Mag. 11, 153 (1965).

18. R. Hultgren, P.D. Desai, D.T. Hawkins, M. Gleiser, K.K. Kelley. Selected Values of the Thermodynamic Properties of Binary Alloys (Am. Soc. Met., 1973) [ISBN: 760175-0].

19. A.B. Bhatia, W.H. Hargrove. Concentration fluctuations and thermodynamic properties of some compound forming binary molten systems. Phys. Rev. B 10, 3186 (1974).

20. R.N. Singh. Short-range order and concentration fluctuations in binary molten alloys. Can. J. Phys. 65, 309 (1987).
21. R.N. Singh, I.S. Jha, D.K. Pandey. Thermodynamics of liquid Mg-Sn alloys. J. Phys. Cond. Matter. 5, 2469 (1993).

22. A. Kumar, S.M. Rafique, placeN. Jha, A.K. Mishra. Structure, thermodynamic, electrical and surface properties of $\mathrm{Cu}-\mathrm{Mg}$ binary alloy: Complex formation model. Physica $B$ 357, 445 (2005).

23. A. Kumar, S.M. Rafique, N. Jha. Study of glass forming tendency of $\mathrm{Ca}-\mathrm{Mg}$ binary alloy and its physical properties: Pseudomolecule formation model. Physica B 373, 169 (2006).

24. A. Kumar, S.M. Rafique, placeN. Jha, T.P. Sinha. Complex formation study of thermodynamical, structural properties and density of states of Al-Mg binary alloy. Physica B 404, 1933 (2009).

25. H.C. Longuet-Higgins. The statistical thermodynamics of multicomponent systems. Proc. Roy. Soc. A 207, 247 (1951).

26. O. Ese, J.A. Reissland. Optimized model potential parameters in metals. J. Phys. F.: Met. Phys. 3, 2066 (1973).

27. R.W. Shaw. Optimum form of a modified Heine-Abarenkov model potential for the theory of simple metals. Phys. Rev. 174, 769 (1968).

28. K. Hiroike. Ornstein-Zernike relation for a fluid mixture with direct correlation functions of finite range. J. Phys. Soc. Japan 27, 1415 (1969).

29. K. Hoshino. Entropy of mixing of compound-forming liquid binary alloys with two types of compound. J. Phys. F: Met. Phys. 12, 1891 (1982).

30. C. Van der Marel, A.B. Van Oosten, W. Geertsma, W. Van der Lugt. The electrical resistivity of liquid $\mathrm{Li}-\mathrm{Sn}, \mathrm{Na}-\mathrm{Sn}$ and $\mathrm{Na}-\mathrm{Pb}$ alloys: Strong effects of chemical interactions. J. Phys. F.: Met. Phys. 12, 2349 (1982).

31. F.W. Dorn, W. Klemm. Das Verhalten der Alkalimetalle zu Halbmetallen. V. Die systeme des antimons mit kalium, rubidium und caesium. Z. Anorg. Allg. Chem. 309, 189 (1961).

32. Y. Waseda. In: Liquid Metals, Edited by R. Evans, D.A. Greenwood (Inst. Phys. of Bristol, 1977), Chap. 2, p. 230 .

33. H. Reiter, H. Ruppersberg, W. Speicher. In: Liquid Metals, 1976. Edited by R. Evans, D.A. Greenwood (Inst. Phys. of Bristol, 1977), p. 133.

Received 26.04.20

А. Кумар, Д.П. Оджа

\section{ВИВЧЕННЯ ТЕРМОДИНАМІЧНИХ}

ВЛАСТИВОСТЕЙ СПЛАВУ Na-Sn I ТРАНСПОРТУ ЕЛЕКТРОНІВ В НЬОМУ

Структуру формули Фабера-Зімана модифіковано із застосуванням поняття "утворення комплексу" з метою вивчення залежності електричного опору сплавів Цінтла від їх складу. Ці сплави аномальні, і поблизу певного значення відношення складових проявляють значне відхилення від поведінки металів.

Ключові слова: змішування, рідини, транспорт електронів, рідкі сплави, термодинамічні властивості. 